Check for updates

Cite this: RSC Adv., 2021, 11, 36310

Received 30th August 2021

Accepted 31st October 2021

DOI: 10.1039/d1ra06527k

rsc.li/rsc-advances

\section{Sulfhydryl functionalized carbon quantum dots as a turn-off fluorescent probe for sensitive detection of $\mathrm{Hg}^{2+} \uparrow$}

\author{
Wei Yao, (D) a Yingchen Hua, ${ }^{b}$ Zhihong Yan, ${ }^{a}$ Chunxian Wu, ${ }^{\text {tb }}$ Feiyan Zhou*c \\ and Yi Liu (D)*abc
}

Mercury ion $\left(\mathrm{Hg}^{2+}\right)$ is one of the most toxic heavy metal ions and lowering the detection limit of $\mathrm{Hg}^{2+}$ is always a challenge in analytical chemistry and environmental analysis. In this work, sulfhydryl functionalized carbon quantum dots (HS-CQDs) were synthesized through a one-pot hydrothermal method. The obtained HS-CQDs were able to detect mercury ions $\mathrm{Hg}^{2+}$ rapidly and sensitively through fluorescence quenching, which may be ascribed to the formation of nonfluorescent ground-state complexes and electron transfer reaction between $\mathrm{HS}-\mathrm{CQDs}$ and $\mathrm{Hg}^{2+}$. A modification of the HS-CQD surface by $-\mathrm{SH}$ was confirmed using Fourier transform infrared spectroscopy (FTIR) and X-ray photoelectron spectroscopy (XPS). The HS-CQDs sensing system obtained a good linear relationship over a $\mathrm{Hg}^{2+}$ concentration ranging from $0.45 \mu \mathrm{M}$ to $2.1 \mu \mathrm{M}$ with a detection limit of $12 \mathrm{nM}$. Delightfully, the sensor has been successfully used to detect $\mathrm{Hg}^{2+}$ in real samples with satisfactory results. This means that the sensor has the potential to be used for testing actual samples.

\section{Introduction}

As we all know, with the development of industry and economy, various heavy metal ions have caused relatively serious pollution threatening human health and ecosystems. ${ }^{1,2}$ Mercury ion $\left(\mathrm{Hg}^{2+}\right)$ is one of the most toxic heavy metal ions. It easily accumulates in the human body through the food chain and can cause a series of health problems, such as lung and kidney function damage, chest pain, dyspnea, nervous system damage, and even cancer. ${ }^{3,4}$ Therefore, it is of great significance to establish a highly selective and sensitive detection method for $\mathrm{Hg}^{2+}$. There are many traditional methods for the determination of $\mathrm{Hg}^{2+}$, such as atomic absorption spectrometry (AAS), ${ }^{5}$ anodic stripping voltammetry (ASV), ${ }^{6}$ inductively coupled plasma atomic emission spectrometry (ICP-AES), ${ }^{7}$ inductively coupled plasma mass spectrometry (ICP-MS), ${ }^{8}$ liquid chromatograph-mass spectrometry (LC-MS) ${ }^{9}$ and other sophisticated methods. However, expensive and complex instruments, a lengthy process and procedures for pre-treatment of complex samples all limit the application of these methods. There have been many novel sensors developed in recent years for

${ }^{a}$ College of Pharmacy, Guangdong Pharmaceutical University, Guangzhou 510000, China.E-mail: Liuyi915@126.com

${ }^{b}$ School of Pharmaceutical and Chemical Engineering, Guangdong Pharmaceutical University, Zhongshan 528400, China

'Guangzhou Baiyunshan Weiyi Industrial Co., Ltd, Guangzhou 510000, China

$\dagger$ Electronic supplementary information (ESI) available: Part of XPS spectrum, UV spectrum and fluorescence spectrum. See DOI: 10.1039/d1ra06527k measuring $\mathrm{Hg}^{2+}$ based on functionalized multi-walled carbon nanotubes, ${ }^{10}$ metal nanoparticles ${ }^{11,12}$ and semiconductor quantum dots (QDs). ${ }^{13}$ Semiconductor QDs have also been widely investigated and used as fluorescent probes due to their unique optical properties and surface modification. Such as, Kumagai et al. modified the MOF material on the surface of QDs by dropping metal-organic frameworks (MOFs) precursors into the quantum dot solution and then the ligands were exchanged for pyridine derivatives. ${ }^{14}$ However, general QDs modification methods involve complicated procedures and expensive reagents. But other than that, the requirement of multiple preprocessing steps, harsh synthetic conditions and expensive raw materials all limit the widespread application of these methods.

Recently, carbon quantum dots (CQDs), a new type of fluorescent nanoparticle, has attracted great interest from researchers. CQDs are usually spherical, have a particle size below $10 \mathrm{~nm}$, and emit fluorescence that is strong and stable. $^{15,16}$ Compared with organic dyes, quantum dots and precious metal nanoparticles, CQDs have many advantages, including good water solubility, excellent photostability, great biocompatibility and low toxicity, which make carbon quantum dots can be effectively used in the practical applications of chemical sensors and biosensors. ${ }^{17,18}$ In the above applications, it has been demonstrated surface modification and heteroatomic doping can enhance the efficiency of CQDs. ${ }^{19,20}$ Gui et al. combined the red carbon dots (CDs) modified with amino groups on the surface with the blue silicon dots (SiDs) modified with carboxyl groups on the surface by activation coupling of carbondiimide. $^{21}$ According to some researches, these 
fluorescence sensors are characterized by their sensitivity and selectivity largely due to the affinity of active groups (such as amino and sulfhydryl) on the CQDs surface for the ions they detect. $^{22-24}$

Hence, because meso-2,3-dimercaptosuccinic acid is rich in sulfhydryl groups and able to do react with citric acid at high temperature and pressure, the meso-2,3-dimercaptosuccinic acid was used as the sulfur source to synthesize successfully the sulfhydryl functionalized carbon quantum dots (HS-CQDs). This one-pot preparation route is very easy, green and ecofriendly and was successful at introducing sulfhydryl functional groups. Compared with other functional group modification methods, this approach does not require either sophisticated equipment or complex procedures. Because of the chelation between -SH and $\mathrm{Hg}^{2+}, 25$ the prepared HS-CQDs were developed to high selectively detect $\mathrm{Hg}^{2+}$ with high accuracy by a novel fluorescence "turn-off" model (Scheme 1). In the presence of ultraviolet light, the prepared HS-CQDs emitted blue fluorescence with high quantum yield (QY) (15.8\%), excellent water solubility and stability. In addition, the possible mechanism of detecting $\mathrm{Hg}^{2+}$ was also elucidated. The developed $\mathrm{Hg}^{2+}$ sensor based on HS-CQDs shows satisfactory detection limit $(\mathrm{LOD}=12 \mathrm{nM})$ and concentration ranges under ideal experimental conditions. To the best of our knowledge, this is lower than most other carbon-based fluorescent probes. What's more important, the results of recovery test showed that the "turn-off" fluorescence reported exhibits high applicability to systems and can be used for the detection of $\mathrm{Hg}^{2+}$ in the environment.

\section{Experimental}

\subsection{Materials and characterization}

Citric acid (99.5\%) was purchased from Macklin Biochemical Co., Ltd (Shanghai, China). meso 2,3-Dimercaptosuccinic acid (98\%) and ammonia (25-28\%) were purchased from Shanghai Lin En Technology Development Co., Ltd. (Shanghai, China). $\mathrm{HgCl}_{2}$ was purchased from Zibo Huatong Chemical Reagent Co., Ltd. (Shandong, China). The metal salts used, including $\mathrm{CdCl}_{2}, \mathrm{ZnSO}_{4}, \mathrm{KCl}, \mathrm{NaCl}, \mathrm{BaCl}_{2} \cdot 2 \mathrm{H}_{2} \mathrm{O}, \mathrm{CuCl}_{2} \cdot 2 \mathrm{H}_{2} \mathrm{O}, \mathrm{MgCl}_{2}-$ $\cdot 6 \mathrm{H}_{2} \mathrm{O}, \mathrm{NiCl}_{2} \cdot 6 \mathrm{H}_{2} \mathrm{O}, \mathrm{MnSO}_{4} \cdot \mathrm{H}_{2} \mathrm{O}, \mathrm{CaCl}_{2}, \mathrm{~Pb}\left(\mathrm{NO}_{3}\right)_{2}, \mathrm{FeCl}_{2}$,
$\mathrm{FeCl}_{3} \cdot 6 \mathrm{H}_{2} \mathrm{O}, \mathrm{AgNO}_{3}$ and $\mathrm{CrCl}_{3} \cdot 6 \mathrm{H}_{2} \mathrm{O}$ were of analytical puritygrade and used without any further purifications. Ultrapure water was produced with a Ultrapure Water System (Nanjing QuanKun Bio-technology Co., Ltd, China) and used throughout the experiments.

UV-visible absorption (UV-Vis) spectrums were measured by using a A11665 UV-Vis spectrophotometer (Shimadzu, Japan). Fourier transform infrared spectroscopy (FTIR) spectrum were performed on a IRAffinity-1 spectrophotometer (Shimadzu, Japan). The fluorescence measurements were recorded using an LF-1804005 fluorescence spectrophotometer (Thermo Fisher Scientific, China). The slit width was set at $5 \mathrm{~nm}$ for both excitation and emission. Transmission electron microscopy (TEM) images were measured by $\mathrm{H}-7650$ (HITACHI, Japan). X-ray photoelectron spectroscopy (XPS) were performed on ESCALAB 250 XI (Thermo Fisher Scientific, China). X-ray diffractometer (XRD) pattern was measured by D/max-2200/PC (Rigaku Corporation, Japan).

\subsection{Synthesis of HS-CQDs}

HS-CQDs were synthesized by a one-pot synthetic method. The $1 \mathrm{~g}$ citric acid and $0.68 \mathrm{~g}$ meso 2,3-dimercaptosuccinic acid were placed into a clean, dry beaker containing $25 \mathrm{~mL}$ of ultrapure water and $10 \mathrm{~mL}$ ammonia was added under rapid stirring to make meso 2,3-dimercaptosuccinic acid sufficiently dissolved. Afterward, the mixture was heated at $150{ }^{\circ} \mathrm{C}$ for 180 minutes in a Teflon-lined autoclave $(40 \mathrm{~mL})$. Cooling until room temperature is reached, the above aqueous solution was bag (1000 Da) for $48 \mathrm{~h}$ to remove raw materials. After dialysis, the solution was filtered through $0.22 \mu \mathrm{m}$ membrane filter to remove the sediment and frozen drying. Finally, the purified HS-CQDs were brown powder and prepared for subsequent use.

\subsection{Fluorescence QY measurement}

The measurement method of fluorescence QY was based on previous studies. ${ }^{26} \mathrm{~A}$ fraction of the reference substance quinine sulfate $(\mathrm{QY}=0.577)$ was dissolved in $0.05 \mathrm{~N}$ sulfuric acid solution as a reference substance, and the emission peak integral area and UV absorbance of the substance to be tested and

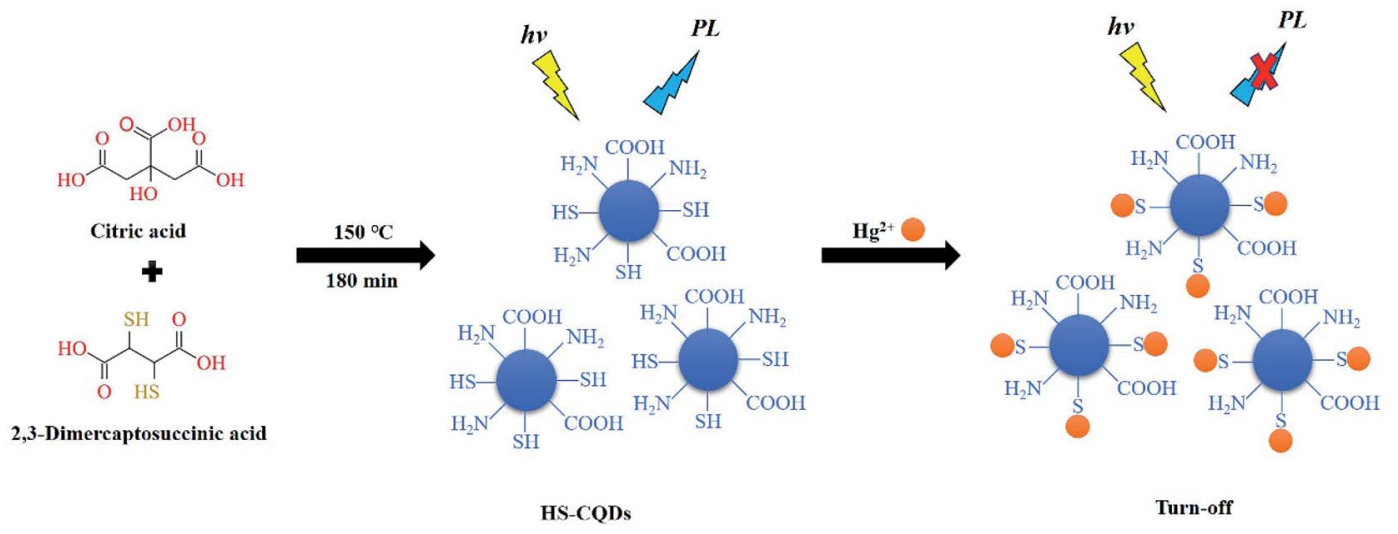

Scheme 1 Schematic presentation of the synthesis of HS-CQDs and the application for $\mathrm{Hg}^{2+}$ detection. 
quinine sulfate were measured at $350 \mathrm{~nm}$ (the UV absorbance remained below 0.05 ). The quantum yield was calculated by the following formula (1):

$$
\mathrm{QY}_{\mathrm{s}}=\mathrm{QY}_{\mathrm{r}}\left(F_{\mathrm{s}} / F_{\mathrm{r}}\right)\left(A_{\mathrm{r}} / A_{\mathrm{s}}\right)\left(\eta_{\mathrm{s}} / \eta_{\mathrm{r}}\right)^{2}
$$

Specially, QY is the quantum yield, $F$ symbolizes the fluorescence emission peak area, $\eta$ is the refractive index of the solvent, and $A$ is the absorbance at the excitation wavelength. The subscript " $r$ " refers to standard and "s" refers to the sample.

\subsection{Detection of $\mathbf{H g}^{2+}$}

The sensing system of $\mathrm{Hg}^{2+}$ were prepared by mixing $4800 \mu \mathrm{L}$ of ultrapure water, $100 \mu \mathrm{L}$ of HS-CQDs $\left(1.0 \mathrm{mg} \mathrm{mL}^{-1}\right)$ and $100 \mu \mathrm{L}$ of $\mathrm{Hg}^{2+}$ with different concentrations. Kept the total volume at $4 \mathrm{~mL}$ and the final concentration of HS-CQDs was $25 \mu \mathrm{g} \mathrm{mL}{ }^{-1}$. After reacting at room temperature for $1 \mathrm{~min}$, the fluorescence spectra were recorded at the excitation wavelength of $350 \mathrm{~nm}$. The linear regression equation was obtained from fluorescence quench factor $\left(1-F / F_{0}\right)$ versus the concentrations of $\mathrm{Hg}^{2+}$ data. To test the selectivity for the sensor toward $\mathrm{Hg}^{2+}$, different metal ions $\left(\mathrm{Pb}^{2+}, \mathrm{Cd}^{2+}, \mathrm{Cr}^{3+}, \mathrm{Zn}^{2+}, \mathrm{Cu}^{2+}, \mathrm{Mn}^{2+}, \mathrm{Ca}^{2+}, \mathrm{Mg}^{2+}, \mathrm{Ba}^{2+}, \mathrm{Ni}^{2+}\right.$, $\mathrm{Fe}^{2+}, \mathrm{Fe}^{3+}, \mathrm{Ag}^{+}, \mathrm{K}^{+}, \mathrm{Na}^{+}$) with the concentration of $10 \mu \mathrm{M}$ were added to $25 \mu \mathrm{g} \mathrm{mL}^{-1}$ HS-CQDs solution. For purpose of assessment the anti-interference of the sensor, $10 \mu \mathrm{M} \mathrm{of} \mathrm{Hg}^{2+}$ and 13 kinds of metal ions with the concentration of $10 \mu \mathrm{M}$ were dripped into HS-CQDs solution.

\subsection{Detection of $\mathrm{Hg}^{2+}$ in real sample}

In order to investigate the practicality of the sensor, the HSCQDs sensing system was used for the detection of $\mathrm{Hg}^{2+}$ in lake water and waste water samples. These samples were come from the artificial lake and living area of the Zhongshan campus of Guangdong Pharmaceutical University. Then, the lake water and waste water were filtered through a $0.22 \mu \mathrm{m}$ microporous membrane and centrifuged at $8000 \mathrm{rpm}$ for $10 \mathrm{~min}$ to remove the particulate matter. Then $1 \mathrm{~mL}$ obtained water samples was added to the mixed solutions with $100 \mu \mathrm{L}$ of HS-CQDs $(1 \mathrm{mg}$ $\left.\mathrm{mL}^{-1}\right)$ and $100 \mu \mathrm{L}$ of different concentrations of $\mathrm{Hg}^{2+}(0.50 \mu \mathrm{M}$, $1.0 \mu \mathrm{M}$ and $2.0 \mu \mathrm{M}$ ) to obtain fluorescence intensities as the proposed method. Each experiment was done three times in parallel.

\section{Results and discussion}

\subsection{Characterization of HS-CQDS}

To explore the surface status and size of HS-CQDs, several characterizations were carried out. As shown in Fig. 1a, the TEM image showed that HS-CQDs were well monodispersed and the morphology of HS-CQDs was spherical. The distributions of particle sizes (Fig. 1b) shows that the HS-CQDs were an average particle size of $\sim 3.5 \mathrm{~nm}$ in the range of $2.75-4.55 \mathrm{~nm}$. The XRD spectrum of the HS-CQDs was shown in Fig. 2a. The sharp peak at around $27^{\circ}$ was attributed to the (002) peak of graphite, which means the HS-CQDs have a graphitic structure. ${ }^{27,28}$ FTIR spectrum of HS-CQDs was shown in Fig. 2b and exhibited characteristic peaks of surface functional groups of HS-CQDs. The peak at $3406 \mathrm{~cm}^{-1}$ was indicated the stretching vibration of the $\mathrm{O}-\mathrm{H}$ bond, and the peak at $3175 \mathrm{~cm}^{-1}$ was designated as the stretching vibration of the $\mathrm{N}-\mathrm{H}$ bond of primary amine. The peak of $2510 \mathrm{~cm}^{-1}$ was indicated $\mathrm{S}-\mathrm{H}$ stretching vibration, ${ }^{29}$ suggesting that the sulfhydryl group has been successfully modified to HS-CQDs. The two peaks locating to $1585 \mathrm{~cm}^{-1}$ and $1400 \mathrm{~cm}^{-1}$ represented the stretching vibration of the $\mathrm{C}=\mathrm{O}$ and $\mathrm{C}-\mathrm{O}$, which was due to the carboxylate in the surface of HSCQDs. And the peak of $1262 \mathrm{~cm}^{-1}$ was attributed to $\mathrm{C}-\mathrm{N}$ stretching vibrations of amide. The FTIR spectrum demonstrated that the HS-CQDs were successfully functionalized with sulfhydryl, amino and carboxylate. ${ }^{30,31}$

To further explore the functional groups and chemical composition of HS-CQDs, the XPS spectra was investigated. The full scan of the XPS spectrum shown in Fig. 2c showed four different typical peaks at $284.8 \mathrm{eV}, 531.6 \mathrm{eV}, 401.1 \mathrm{eV}$ and $163.3 \mathrm{eV}$ attributed to carbon (C1s), oxygen (O1s), nitrogen (N1s) and sulfur (S2p) respectively, and their contents were $48.95 \%$, $43.19 \%, 5.17 \%$ and $2.69 \%$, respectively. The high-resolution spectrum of C1s spectrum (Fig. S1a $\dagger$ ) was deconvoluted into four peaks at $288.4 \mathrm{eV}, 286.5 \mathrm{eV}, 284.9 \mathrm{eV}$ and $284.7 \mathrm{eV}$, which were attributed to $\mathrm{C}-\mathrm{O}-\mathrm{C}, \mathrm{C}=\mathrm{O}, \mathrm{C}-\mathrm{N}$ and $\mathrm{C}-\mathrm{S}$. The highresolution spectrum of $\mathrm{O} 1 \mathrm{~s}$ spectrum (Fig. $\mathrm{S} 1 \mathrm{~b} \dagger$ ) presented two peaks at $532 \mathrm{eV}$ and $531.1 \mathrm{eV}$, which were owing to $\mathrm{C}-\mathrm{OH} / \mathrm{C}-$ (a)

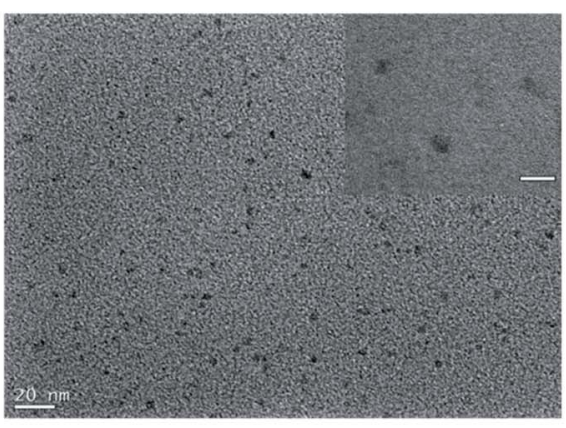

(b)

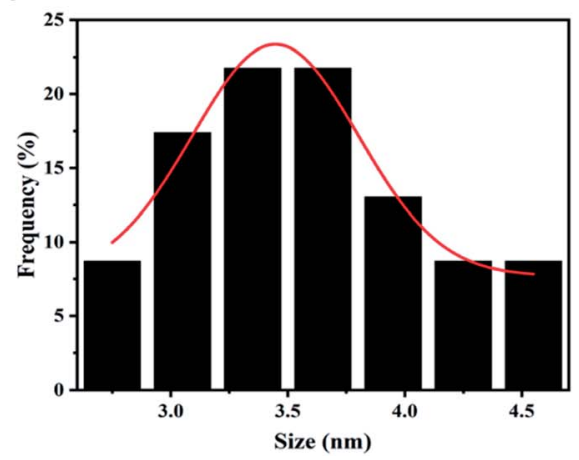

Fig. 1 (a) TEM of HS-CQDs (inset: the bar: $10 \mathrm{~nm}$ ). (b) Size distribution histogram of HS-CQDs. 
(a)

$\mathrm{O}-\mathrm{C}$ and $\mathrm{C}=\mathrm{O}$. The high resolution spectrum of N1s spectrum (Fig. S1c $\dagger$ ) was deconvoluted into three peaks at $401.2 \mathrm{eV}, 400.5 \mathrm{eV}$ and $399.5 \mathrm{eV}$, corresponding to $\mathrm{N}-\mathrm{H}, \mathrm{N}-\mathrm{C}$ and $\mathrm{C}-\mathrm{N}-\mathrm{C}$, respectively. The high-resolution spectrum of S2p spectrum (Fig. 2d) was divided into three peaks at $168.1 \mathrm{eV}$, $164.3 \mathrm{eV}$ and $163.2 \mathrm{eV}$, which derived from the C-S covalent bonds of the thiophene-S for their spin-orbit couplings and sulfate or sulfonate. ${ }^{24,32}$ On the basis of the above results, both FTIR and XPS characterization methods have proved the successful functionalization of HS-CQDs with $-\mathrm{COO}^{-},-\mathrm{NH}_{2}$ and $-\mathrm{SH}$.

\subsection{Optical properties}

The HS-CQDs solution was pellucid light yellow in natural light and displayed bluish violet under $365 \mathrm{~nm}$ UV light (inset in Fig. 3a). In order to further explore the optical properties of HSCQDs, HS-CQDs was characterized by UV-vis absorption spectrum and fluorescence spectrum. As shown in Fig. 3a, the weak peak at $\sim 265 \mathrm{~nm}$ was derived from the $\pi-\pi^{*}$ transition of $\mathrm{C}=$ C. ${ }^{33}$ And there was a strong obviously absorption peak at $\sim 338 \mathrm{~nm}$ attributed to $\mathrm{n}-\pi^{*}$ transition and $\mathrm{n}-\sigma^{*}$ transition, which were relevant to the oxygen-containing functional groups (b)

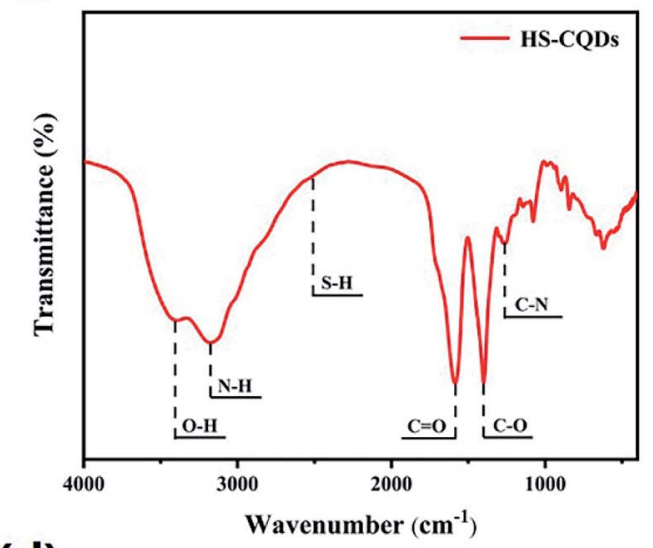

(d)

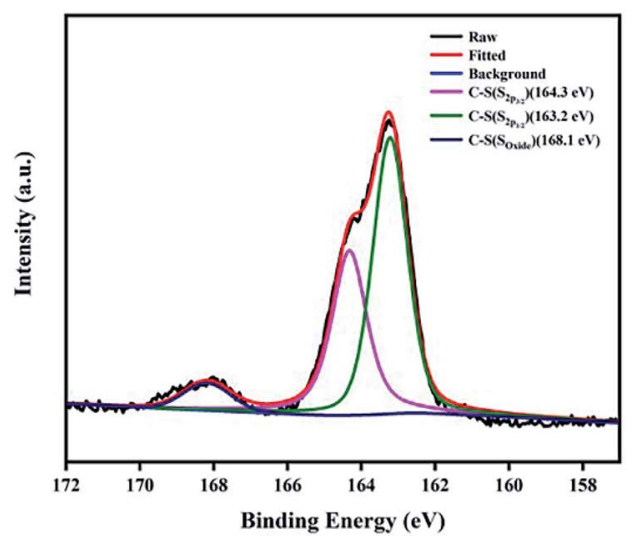

Fig. 2 (a) XRD pattern of HS-CQDs. (b) FTIR of HS-CQDs. (c) XPS survey spectrum of HS-CQDs. (d) High resolution XPS spectra of S2p of HSCQDs.

such as $\mathrm{C}=\mathrm{O}$ and heteroatom groups like $-\mathrm{SH},-\mathrm{OH}$ and $-\mathrm{NH}_{2} \cdot{ }^{34,35}$ The corresponding band gaps of $3.6 \mathrm{eV}$ for HS-CQDs were calculated by using equation $E_{\mathrm{g}}=1240 / \lambda$, mainly attributed to the electronic transitions from the valence. ${ }^{36,37}$ As a result of these functional groups, HS-CQDs are more soluble in aqueous solutions and their surface states are changed to enhance their QY. Therefore, with the QY of quinine sulfate as the reference, the QY of HS-CQDs was about $15.8 \%$ at the excitation wavelength of $350 \mathrm{~nm}$. This QY of the HS-CQDs prepared in our work is higher than those previous researches reported, ${ }^{38,39}$ which illustrates the extraordinary fluorescent properties and good application prospect of the HS-CQDs in our work.

To inquire into the fluorescent properties of HS-CQDs, the photoluminescence (PL) emission behavior of the HS-CQDs under different excitation wavelengths was investigated. As can be seen from the fluorescence spectrum (Fig. 3b), the fluorescence intensity presented a change law of first increasing and then decreasing with the excitation wavelength increasing from $320 \mathrm{~nm}$ to $380 \mathrm{~nm}$. Moreover, the maximum photoluminescence peaks showed a significant shift. This clearly shows the excitation-dependent PL behavior of HS-CQDs, which was correspond to the properties of carbon quantum dots by 
(a)

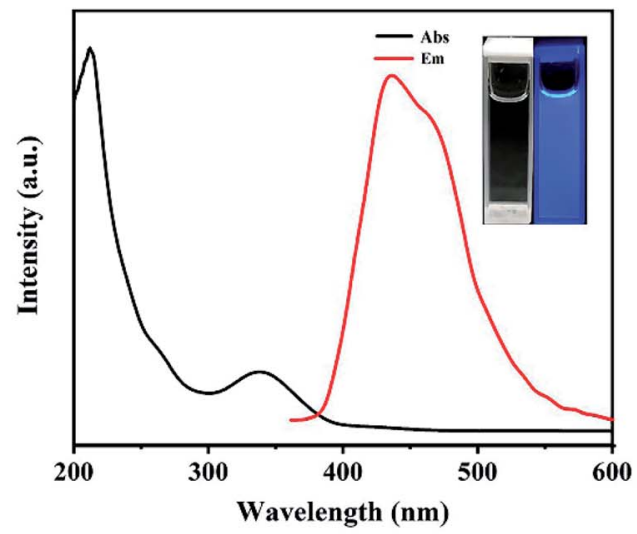

(b)

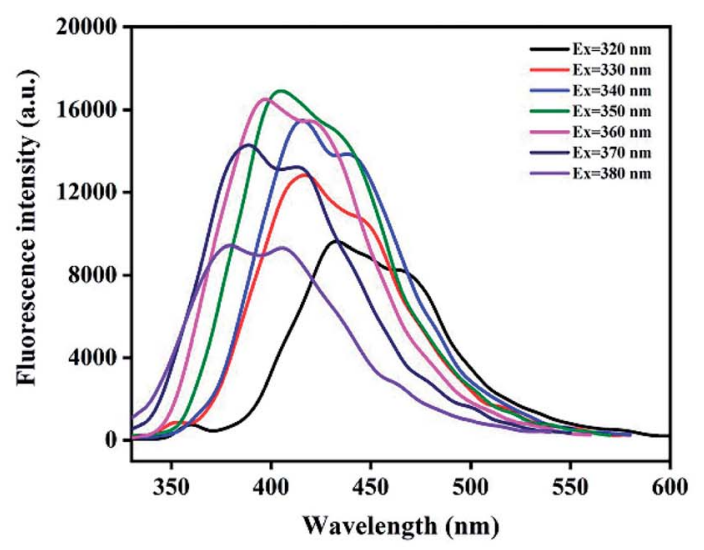

Fig. 3 (a) UV-Vis absorption spectrum and fluorescence spectrums $\left(\lambda_{\text {ex }}=350 \mathrm{~nm}\right.$ ) (inset: photographs of HS-CQDs under the radiation of (left) visible light and (right) $365 \mathrm{~nm}$ UV light). (b) Fluorescence spectra of HS-CQDs solutions $\left(25 \mu \mathrm{g} \mathrm{mL}^{-1}\right.$ ) with different excitation wavelengths from $320 \mathrm{~nm}$ to $380 \mathrm{~nm}$.

previous publications. ${ }^{3,40}$ This excitation wavelength-dependent property may be caused by the selection of nanoparticles based on their size (quantum effect) or different emissive traps on the surface of HS-CQDs. ${ }^{26,41}$ When the excitation wavelength is $350 \mathrm{~nm}$, the peak value of fluorescence emission spectrum reaches the maximum. Hence, the wavelength of $350 \mathrm{~nm}$ was intended to be the best excitation wavelength.

Then, the effects of time, temperature, ionic strength and $\mathrm{pH}$ value on the fluorescence stability of HS-CQDs were evaluated. As shown in Fig. S2 and S3, $\uparrow$ the color of HS-CQDs solutions (a)

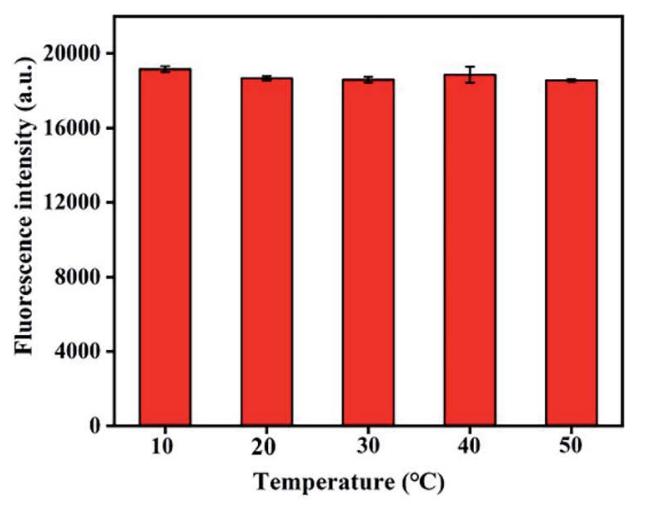

(c)

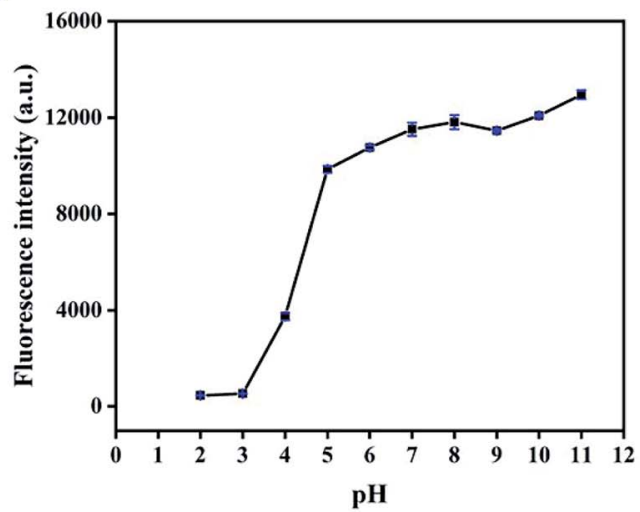

(b)

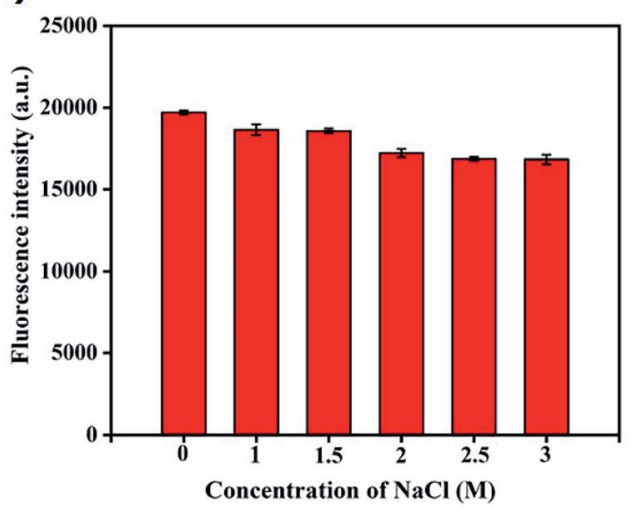

(d)

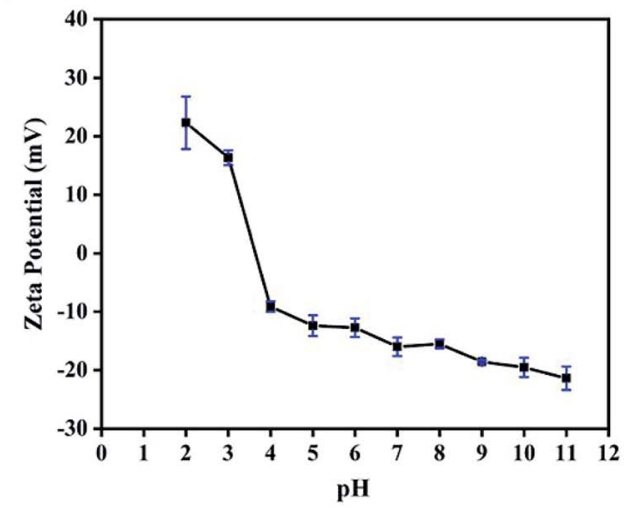

Fig. 4 The effect of (a) temperature $\left(10-50{ }^{\circ} \mathrm{C}\right)$, (b) ionic strength in $\mathrm{NaCl}$ aqueous solution (1-3 $\left.\mathrm{M}\right)$ and (c) pH (2-11) on the fluorescence intensity of HS-CQDs $\left(25 \mu \mathrm{g} \mathrm{mL}{ }^{-1}\right.$ ). (d) The effect of $\mathrm{pH}$ on the zeta potential of HS-CQDs $\left(25 \mu \mathrm{g} \mathrm{mL}{ }^{-1}\right.$ ). $\lambda_{\text {ex }}=350 \mathrm{~nm}$. 
Table 1 Comparison of the proposed methods with previous methods employed for $\mathrm{Hg}^{2+}$ detection based on carbon quantum dots

\begin{tabular}{llll}
\hline Methods & Linear range & Detection limit \\
\hline HS-CQDs as "turn-off" fluorescence probe & $0.45-2.1 \mu \mathrm{M}$ & $12 \mathrm{nM}$ & Ref. \\
Au/N-CQDs as"on-off-on" fluorescence probe & $0-41.86 \mu \mathrm{M}$ & $0.118 \mu \mathrm{M}$ & This work \\
N-CQDs as “off-on" fluorescence probe & $0-18 \mu \mathrm{M}$ & $83.5 \mathrm{nM}$ & 23 \\
CDs as fluorescence probe & $0-80 \mu \mathrm{M}$ & $201 \mathrm{nM}$ & 46 \\
N-CQDs as fluorescence probe & $0.001-0.1 \mu \mathrm{M}$ & $5.3 \mathrm{nM}$ & 47 \\
BN-CDs as fluorescence probe & $5-175 \mu \mathrm{M}$ & $2.8 \mu \mathrm{M}$ & 41
\end{tabular}

hardly changed and still showed a very light yellowish color after being placed for $8 \mathrm{~h}$ and $24 \mathrm{~h}$, and the fluorescence of HSCQDs solutions hardly changed. These results indicated that HS-CQDs has good stability in aqueous solution. Further, it is clear that the fluorescence intensity of HS-CQDs kept stable when the temperature increased from 10 to $50{ }^{\circ} \mathrm{C}$ and the concentrations of $\mathrm{NaCl}$ aqueous solution ranged from 1 to $3 \mathrm{M}$, which indicated that temperature and ion strength had almost no effect on the character of HS-CQDs (Fig. 4a and b). As shown in Fig. 4c and d, the fluorescence intensity of HS-CQDs had sharply changed and the zeta potential had changed from positive to negative potentials with the $\mathrm{pH}$ changing from 2 to 11 , which is due to the extensive protonation-deprotonation of the amide group and of the HS-CQDs. ${ }^{42}$ This suggests that the HS-CQDs is $\mathrm{pH}$ responsive, which is similar to other CQDs reported by previous research..$^{43,44}$

\subsection{Detection of $\mathbf{H g}^{2+}$}

In order to obtain optimal reaction conditions, the effect of $\mathrm{pH}$ and incubation time on the relationship between fluorescence quenching rate $F / F_{0}$ and HS-CQDs $+\mathrm{Hg}^{2+}$ solution was studied. $F_{0}$ and $F$ represented PL intensity of HS-CQDs before and after adding $\mathrm{Hg}^{2+}$, respectively, and the concentrations of HS-CQDs solution and $\mathrm{Hg}^{2+}$ solution were $25 \mu \mathrm{g} \mathrm{mL} \mathrm{m}^{-1}$ and $10 \mu \mathrm{M}$ respectively. As shown in Fig. $\mathrm{S} 4, \dagger$ the PL quenching rate of HSCQDs has fallen substantially in the $\mathrm{pH}$ range of $4-10$, and the reaction was complete within 1 minute, which indicated that this fluorescent probe can quickly complete the detection of $\mathrm{Hg}^{2+}$ under harsh conditions. Therefore, the best experimental conditions were found when the $\mathrm{Hg}^{2+}$ detection was carried out in the $\mathrm{pH}=7$ ultrapure water after incubating with HS-CQDs for $1 \mathrm{~min}$.

To evaluate the sensitivity of the HS-CQDs for $\mathrm{Hg}^{2+}$, the detection limit for $\mathrm{Hg}^{2+}$ detection was explored. As shown in the Fig. 5a, the PL intensity was reduced gradually with gradients of $\mathrm{Hg}^{2+}(0-10 \mu \mathrm{M})$ dripped into the HS-CQDs solution $(25 \mu \mathrm{g}$ $\mathrm{mL}^{-1}$ ), implying that the PL intensity of the HS-CQDs is very sensitive to $\mathrm{Hg}^{2+}$. The plot of PL quenching rate against concentrations of $\mathrm{Hg}^{2+}$ is presented in Fig. 5b. As illustrated in the inset of Fig. 5b, an excellent linear relationship with the concentration of $\mathrm{Hg}^{2+}$ in the range of $0.45-2.1 \mu \mathrm{M}$ is observed. The linear equation was $F / F_{0}-1=0.3824 C_{\mathrm{Hg}^{2+}}-0.08390$ with a good linear correlation (the correlation coefficient $\left(R^{2}\right)$ of 0.9943). The limit of detection (LOD) for $\mathrm{Hg}^{2+}$ based on a signalto-noise ratio $(\mathrm{S} / \mathrm{N})$ of 3 was approximately $12 \mathrm{nM}$, which was extremely lower than other fluorescent probes based on carbon materials (Table 1 ).

For the sake of the selectivity of the HS-CQDs for $\mathrm{Hg}^{2+}$, the PL intensity variations before and after adding $10 \mu \mathrm{M}$ of metal ions, including $\mathrm{Hg}^{2+}, \mathrm{Cd}^{2+}, \mathrm{Zn}^{2+}, \mathrm{K}^{+}, \mathrm{Na}^{+}, \mathrm{Cu}^{2+}, \mathrm{Ni}^{2+}, \mathrm{Pb}^{2+}, \mathrm{Fe}^{2+}, \mathrm{Ba}^{2+}$, $\mathrm{Mg}^{2+}, \mathrm{Mn}^{2+}, \mathrm{Ca}^{2+}, \mathrm{Cr}^{6+}, \mathrm{Fe}^{3+}, \mathrm{Ag}^{+}$and $\mathrm{Cr}^{3+}$ to the HS-CQDS solution were investigated separately. As shown in Fig. 6a, the (a)

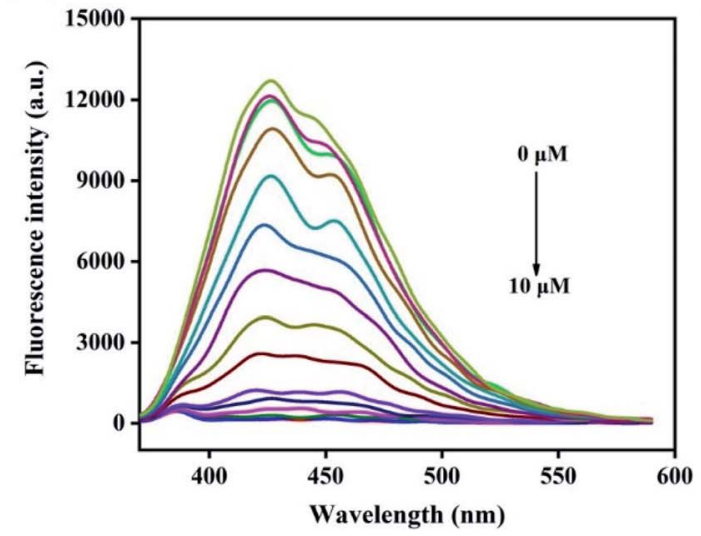

(b)

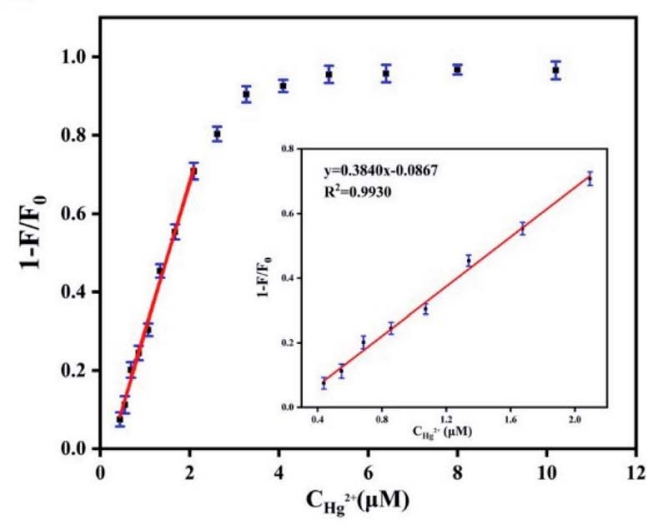

Fig. 5 (a) Emission spectra of HS-CQDs $\left(25 \mu \mathrm{g} \mathrm{mL}{ }^{-1}\right.$ ) with various concentrations of $\mathrm{Hg}^{2+}$ in ultrapure water (pH $=7$ ). $\lambda_{\text {ex }}=350 \mathrm{~nm}$. (b) Relationship between fluorescence intensity variation and $\mathrm{Hg}^{2+}$ concentration ranging from $0 \mu \mathrm{M}$ to $10 \mu \mathrm{M}$. The measurement was carried out in $\mathrm{pH}=7$ ultrapure water containing $25 \mu \mathrm{g} \mathrm{mL} \mathrm{LS}^{-1} \mathrm{HS}$-CQDs. $\lambda_{\mathrm{ex}}=350 \mathrm{~nm}$. 
(a) (b)

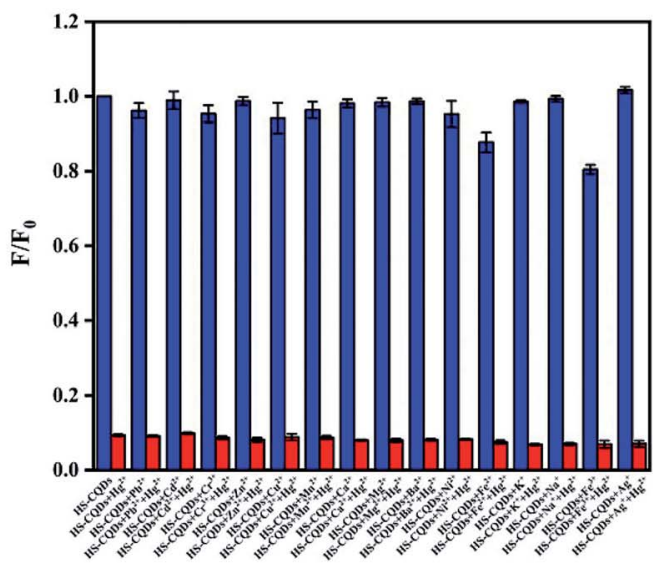

Fig. 6 (a) Selectivity and (b) anti-interference of HS-CQDs for the detection to $\mathrm{Hg}^{2+}$. The measurement was carried out in $\mathrm{pH}=7 \mathrm{ultrapure}$ water containing $25 \mu \mathrm{g} \mathrm{mL}^{-1} \mathrm{HS}-\mathrm{CQDs}$ or $10 \mu \mathrm{M}$ metal ions. $\lambda_{\text {ex }}=350 \mathrm{~nm}$.

PL intensity of HS-CQDs is dramatically decreased (expressed as $\left.F / F_{0}\right)$ only in the presence of $\mathrm{Hg}^{2+}$, and other metal ions caused almost no effect on the fluorescence intensity of the HS-CQDs.
Fig. $6 \mathrm{~b}$ shows the anti-interference performance for HS-CQDs detecting towards $\mathrm{Hg}^{2+}$. The blue columns represented the HS-CQDS solution added with other heavy metal ions, and the (a)

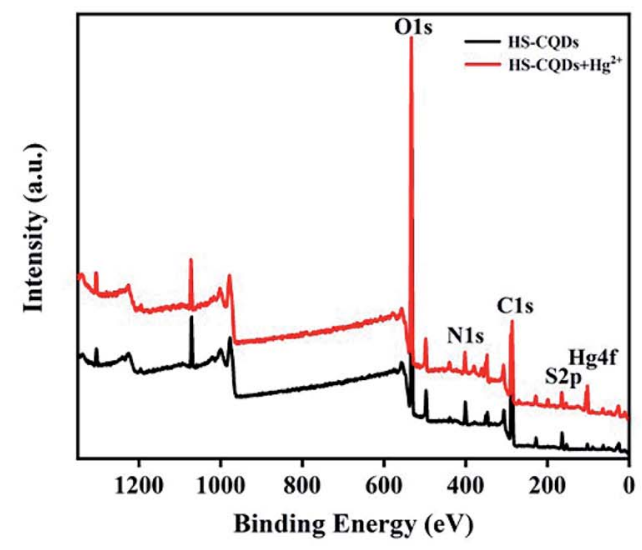

(d)

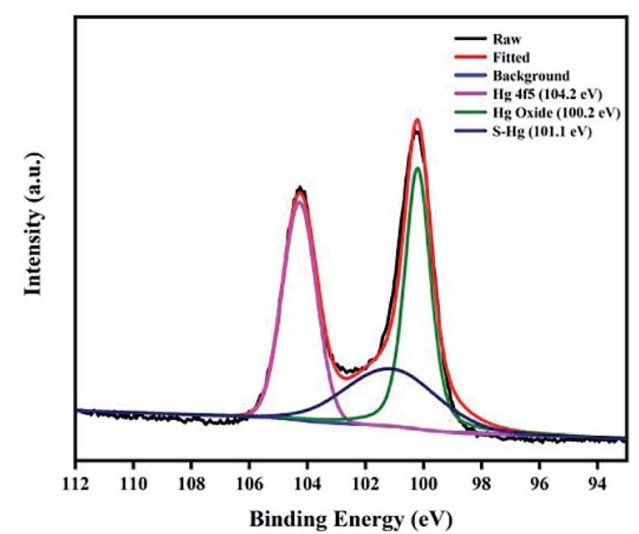

(b)

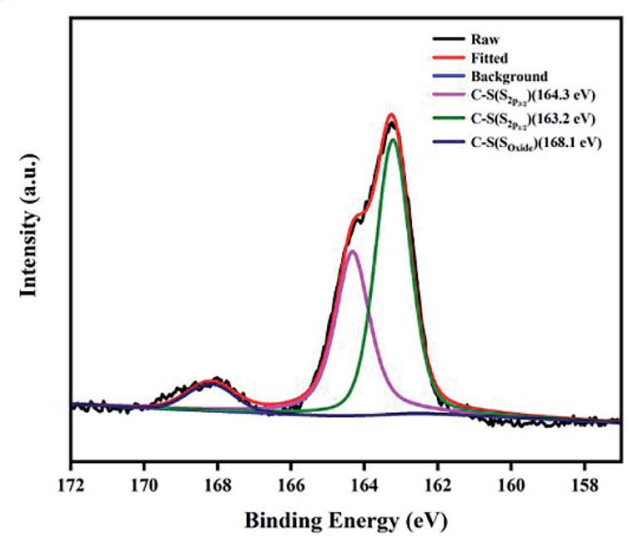

(c)

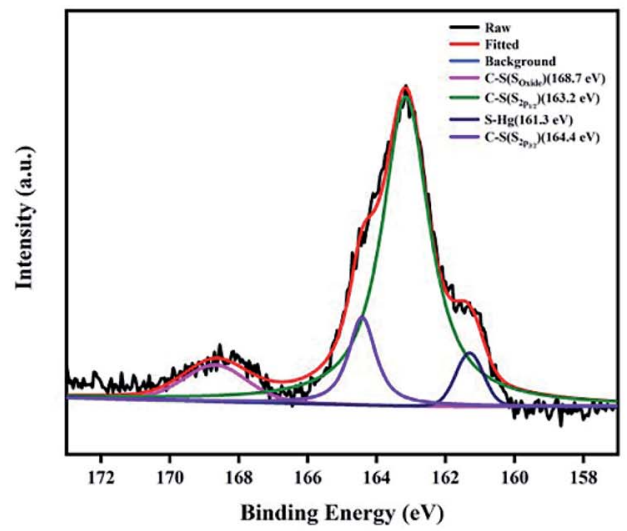

Fig. 7 (a) XPS survey spectrum of the HS-CQDs with or without $\mathrm{Hg}^{2+}$. (b) High resolution XPS spectra of S2p of HS-CQDs. (c) High resolution XPS spectra of S2p of HS-CQDs-Hg complexes. (d) High resolution XPS spectra of Hg4f of HS-CQDs-Hg complexes. 
Table 2 Detection of $\mathrm{Hg}^{2+}$ in real samples $(n=3)$

\begin{tabular}{lllll}
\hline Sample & Add $(\mu \mathrm{M})$ & Found $(\mu \mathrm{M})$ & $\begin{array}{l}\text { Recovery } \\
(\%)\end{array}$ & RSD $(\%)$ \\
\hline \multirow{2}{*}{ Lake water } & 0 & 0.020 & - & 1.4 \\
& 0.50 & 0.51 & 102.6 & 1.6 \\
& 1.0 & 1.1 & 105.7 & 2.0 \\
Waste water & 2.0 & 2.0 & 97.79 & 0.5 \\
& 0.50 & 0.042 & - & 1.7 \\
& 1.0 & 0.52 & 104.0 & 4.1 \\
& 2.0 & 1.1 & 102.1 & 3.6 \\
& & 2.0 & 101.7 & 1.8
\end{tabular}

red columns represented the HS-CQDS solution added with other heavy metal ions and $\mathrm{Hg}^{2+}$. It is clearly indicated that there was no significant effect on the PL intensity of the HSCQDs in the presence of interference metal ions. However, when $\mathrm{Hg}^{2+}$ ions were added to the HS-CQDs solutions, remarkable PL quenching was noticed. From these results we can infer the conclusion that the HS-CQDs have an excellent selectivity in detection of $\mathrm{Hg}^{2+}$.

\subsection{Sensing mechanism of HS-CQDs for $\mathbf{H g}^{2+}$}

$\mathrm{Zu}$ et al. first summarized the various kinds of quenching mechanisms of CQDs. ${ }^{48}$ In general, quenching mechanisms include static quenching, dynamic quenching, Förster resonance energy transfer (FRET) ${ }^{49}$ photoinduced electron transfer $(\mathrm{PET}),{ }^{50}$ surface energy transfer (SET), ${ }^{51}$ dexter energy transfer (DET) and inner filter effect (IFE). ${ }^{52}$ In this work, HS-CQDs detect $\mathrm{Hg}^{2+}$ through the static quenching effect, which is the nonfluorescent ground-state complex that occurs through the interaction between HS-CQDs and $\mathrm{Hg}^{2+} \cdot{ }^{53}$ Fig. S5 $\dagger$ showed that the absorption peak of HS-CQDs at $338 \mathrm{~nm}$ disappeared after dripping $\mathrm{Hg}^{2+}$ into the sensing system. This indicated that the fluorescence quenching may due to the chelation between $\mathrm{Hg}^{2+}$ and one of the groups of $-\mathrm{SH},-\mathrm{COOH}$ and $-\mathrm{NH}_{2}$ on HS-CQDs. The full scan of the XPS spectrum of HS-CQDs and HS-CQDs$\mathrm{Hg}$ complexes was shown in Fig. 7a, and the peak at $100.2 \mathrm{eV}$ of $\mathrm{Hg} 4 \mathrm{f}$ illustrated the chelation between HS-CQDs and $\mathrm{Hg}^{2+}$. The high resolution XPS spectra of S2p of HS-CQDs and HS-CQDs$\mathrm{Hg}$ complexes (Fig. $7 \mathrm{~b}$ and $\mathrm{c}$ ), and $\mathrm{Hg} 4 \mathrm{f}$ of HS-CQDs- $\mathrm{Hg}$ complexes further explained the existence of chelates (Fig. 7d). The peak at $161.3 \mathrm{eV}$ of S2p spectra and the peak at $101.1 \mathrm{eV}$ of $\mathrm{Hg} 4 \mathrm{f}$ spectrum were attributed to $\mathrm{S}-\mathrm{Hg}$, which demonstrated the chelation between $\mathrm{Hg}^{2+}$ and -SH on HS-CQDs.

\subsection{Application to real sample analysis}

In order to evaluate the practicality of this fluorescent probe, the HS-CQDs sensing system was applied to detecting $\mathrm{Hg}^{2+}$ in lake water and waste water. As listed in Table 2, the recoveries of $\mathrm{Hg}^{2+}$ in real samples are ranging from $97.79 \%$ to $105.7 \%$. Further, the relative standard deviations (RSD) of three replicate detections for each sample below 5.0\%, and thus, quite acceptable. It indicated that this fluorescent probe used to detect $\mathrm{Hg}^{2+}$ in real sample is still sensitive. All these studies have shown that this fluorescent probe is an effective, practical, sensitive and selective fluorescent sensor for monitoring $\mathrm{Hg}^{2+}$ levels in real water samples.

\section{Conclusion}

In summary, we first introduced meso-2,3-dimercaptosuccinic acid as sulfur source to synthesize HS-CQDs through a simple, low-consumption and pollution-free one-pot hydrothermal method. The size of particles of HS-CQDs on average is $3.5 \mathrm{~nm}$ with good quantum yield and fluorescence properties. Based on the above characterization results, it can be seen directly that -SH was successfully modified on the surface of CQDs. In addition, the prepared HS-CQDs were used for $\mathrm{Hg}^{2+}$ detection due to their highly selectivity, sensitivity, and effectiveness. Delightedly, it has proved that the fluorescence quenching mechanism of detecting $\mathrm{Hg}^{2+}$ is realized by the formation of nonfluorescent ground-state complexes and electron transfer reaction between $\mathrm{HS}-\mathrm{CQDS}$ and $\mathrm{Hg}^{2+}$, leading to the static fluorescence quenching of HS-CQDs. In the fluorescence "turnoff" processes, this fluorescent probe obtained a good linear relationship over $\mathrm{Hg}^{2+}$ concentration ranging from $0.45 \mu \mathrm{M}$ to $2.1 \mu \mathrm{M}$ with a detection limit of $12 \mathrm{nM}$. The sensor has been successfully used to detect $\mathrm{Hg}^{2+}$ in real samples with satisfactory results. Furthermore, due to the fact that the synthesis method of HS-CQDs was straightforward, rapid, time-saving and cost-effective, it has provided a possibility for the practical application of $\mathrm{Hg}^{2+}$ detection with high sensitivity and selectivity in the future.

\section{Conflicts of interest}

There are no conflicts to declare.

\section{Acknowledgements}

We thank the 2021 Guangdong University Students' Innovation and Entrepreneurship Practice Project (202110573003), 2019 Guangdong University Students' Entrepreneurship Practice Training Project (201910573034) and 2021 China University Student Innovation Training Project (202110573009) for financial support. We also thank Guangdong Province Engineering Technology Centre for Molecular Probe and Bio-medicine Imaging for the technical support.

\section{References}

1 L. A. Malik, A. Bashir, A. Qureashi and A. H. Pandith, Environ. Chem. Lett., 2019, 17, 1495-1521.

2 Q. Yang, Z. Li, X. Lu, Q. Duan, L. Huang and J. Bi, Sci. Total Environ., 2018, 642, 690-700.

3 Z. Liu, Z. Mo, N. Liu, R. Guo, X. Niu, P. Zhao and X. Yang, J. Photochem. Photobiol., A, 2020, 389, 112255.

4 Z. Liu, Z. Mo, X. Niu, X. Yang, Y. Jiang, P. Zhao, N. Liu and R. Guo, J. Colloid Interface Sci., 2020, 566, 357-368.

5 S. Nazerdeylami and R. Zare-Dorabei, Micro Nano Lett., 2019, 14, 823-827. 
6 N. Li, D. Zhang, Q. Zhang, Y. Lu, J. Jiang, G. L. Liu and Q. Liu, Sens. Actuators, B, 2016, 231, 349-356.

7 I. P. Paktsevanidou, N. Manousi and G. A. Zachariadis, Anal. Lett., 2021, 54, 2227-2238.

8 O. V. Kuznetsova, Y. V. Bychkova and A. R. Timerbaev, Anal. Lett., 2020, 53, 563-573.

9 B. Xie, Y. Wang, D. R. Jones, K. K. Dey, X. Wang, Y. Li, J.-H. Cho, T. I. Shaw, H. Tan and J. Peng, Anal. Chem., 2018, 90, 8538-8545.

10 D. Zhang, Y. Yin and J. Liu, Chem. Speciation Bioavailability, 2017, 29, 161-169.

11 A. R. Ferhan, L. Guo, X. Zhou, P. Chen, S. Hong and D.-H. Kim, Anal. Chem., 2013, 85, 4094-4099.

12 H. Cao, M. Wei, Z. Chen and Y. Huang, Analyst, 2013, 138, 2420-2426.

13 M. Li, X. Zhou, S. Guo and N. Wu, Biosens. Bioelectron., 2013, 43, 69-74.

14 K. Kumagai, T. Uematsu, T. Torimoto and S. Kuwabata, CrystEngComm, 2019, 21, 5568-5577.

15 X. Dong, Y. Su, H. Geng, Z. Li, C. Yang, X. Li and Y. Zhang, J. Mater. Chem. C, 2014, 2, 7477-7481.

16 J. Hou, J. Yan, Q. Zhao, Y. Li, H. Ding and L. Ding, Nanoscale, 2013, 5, 9558-9561.

17 Y. Zhai, Z. Zhu, C. Zhu, J. Ren, E. Wang and S. Dong, J. Mater. Chem. B, 2014, 2, 6995-6999.

18 Y. Song, S. Zhu and B. Yang, RSC Adv., 2014, 4, 27184-27200.

19 H. Fei, R. Ye, G. Ye, Y. Gong, Z. Peng, X. Fan, E. L. G. Samuel, P. M. Ajayan and J. M. Tour, ACS Nano, 2014, 8, 10837-10843.

20 S. Jahan, F. Mansoor, S. Naz, J. Lei and S. Kanwal, Anal. Chem., 2013, 85, 10232-10239.

21 R. Gui, X. Bu, W. He and H. Jin, New J. Chem., 2018, 42, 16217-16225.

22 Z. Cheng, F. Du, L. Sun, L. Jiang, G. Ruan and J. Li, ChemistrySelect, 2019, 4, 2122-2128.

23 H. Huang, Y. Weng, L. Zheng, B. Yao, W. Weng and X. Lin, J. Colloid Interface Sci., 2017, 506, 373-378.

24 W. Wang, Y.-C. Lu, H. Huang, A.-J. Wang, J.-R. Chen and J.-J. Feng, Sens. Actuators, B, 2014, 202, 741-747.

25 R. P. Cosson, Biol. Trace Elem. Res., 1994, 46, 229-245.

26 S. Zhu, Q. Meng, L. Wang, J. Zhang, Y. Song, H. Jin, K. Zhang, H. Sun, H. Wang and B. Yang, Angew. Chem., Int. Ed., 2013, 52, 3953-3957.

27 Y. Kim and J. Kim, Opt. Mater., 2020, 99, 109514.

28 H.-Y. Fang, W.-M. Huang and D.-H. Chen, Nanotechnology, 2019, 30, 365603.

29 Y. Dong, H. Pang, H. B. Yang, C. Guo, J. Shao, Y. Chi, C. M. Li and T. Yu, Angew. Chem., Int. Ed., 2013, 52, 7800-7804.

30 Y. Wang, S.-H. Kim and L. Feng, Anal. Chim. Acta, 2015, 890, 134-142.
31 S. Liao, X. Li, H. Yang and X. Chen, Talanta, 2019, 194, 554562.

32 D. Gu, L. Hong, L. Zhang, H. Liu and S. Shang, J. Photochem. Photobiol., B, 2018, 186, 144-151.

33 G. Xu, Y. Niu, X. Yang, Z. Jin, Y. Wang, Y. Xu and H. Niu, Adv. Opt. Mater., 2018, 6, 1800951.

34 X. Hu, X. An and L. Li, Mater. Sci. Eng., C, 2016, 58, 730-736. 35 F. Yan, Y. Zou, M. Wang, X. Mu, N. Yang and L. Chen, Sens. Actuators, B, 2014, 192, 488-495.

36 M. Humayun, A. Zada, Z. Li, M. Xie, X. Zhang, Y. Qu, F. Raziq and L. Jing, Appl. Catal., B, 2016, 180, 219-226.

37 W. Wang, L. Jing, Y. Qu, Y. Luan, H. Fu and Y. Xiao, J. Hazard. Mater., 2012, 243, 169-178.

38 L. Lin, Y. Wang, Y. Xiao and W. Liu, Microchim. Acta, 2019, 186, 1-7.

39 V. A. Ansi and N. K. Renuka, Sens. Actuators, B, 2018, 264, 6775.

40 A. Iqbal, K. Iqbal, L. Xu, B. Li, D. Gong, X. Liu, Y. Guo, W. Liu, W. Qin and H. Guo, Sens. Actuators, B, 2018, 255, 1130-1138.

41 S. Liu, J. Tian, L. Wang, Y. Luo, J. Zhai and X. Sun, J. Mater. Chem., 2011, 21, 11726-11729.

42 X. Liu, H.-B. Li, L. Shi, X. Meng, Y. Wang, X. Chen, H. Xu, W. Zhang, X. Fang and T. Ding, J. Mater. Chem. C, 2017, 5, 10302-10312.

43 F. Yan, D. Shi, T. Zheng, K. Yun, X. Zhou and L. Chen, Sens. Actuators, B, 2016, 224, 926-935.

44 B. Chen, F. Li, S. Li, W. Weng, H. Guo, T. Guo, X. Zhang, Y. Chen, T. Huang, X. Hong, S. You, Y. Lin, K. Zeng and S. Chen, Nanoscale, 2013, 5, 1967-1971.

45 A. Meng, Q. Xu, K. Zhao, Z. Li, J. Liang and Q. Li, Sens. Actuators, B, 2018, 255, 657-665.

46 J. He, H. Zhang, J. Zou, Y. Liu, J. Zhuang, Y. Xiao and B. Lei, Biosens. Bioelectron., 2016, 79, 531-535.

47 A. Tadesse, M. Hagos, D. RamaDevi, K. Basavaiah and N. Belachew, ACS Omega, 2020, 5, 3889-3898.

48 F. Zu, F. Yan, Z. Bai, J. Xu, Y. Wang, Y. Huang and X. Zhou, Microchim. Acta, 2017, 184, 1899-1914.

49 H. Liu, C. Xu, Y. Bai, L. Liu, D. Liao, J. Liang, L. Liu and H. Han, Spectrochim. Acta, Part A, 2017, 171, 311-316.

50 A. Iqbal, Y. Tian, X. Wang, D. Gong, Y. Guo, K. Iqbal, Z. Wang, W. Liu and W. Qin, Sens. Actuators, B, 2016, 237, 408-415.

51 Y. Chen, M. B. O'Donoghue, Y.-F. Huang, H.-Z. Kang, J. A. Phillips, X.-L. Chen, M. C. Estevez, C.-Y. J. Yang and W.-H. Tan, J. Am. Chem. Soc., 2010, 132, 16559-16570.

52 T. D. Gauthier, E. C. Shane, W. F. Guerin, W. R. Seitz and C. L. Grant, Environ. Sci. Technol., 1986, 20, 1162-1166.

53 S. Wang, H. Chen, H. Xie, L. Wei, L. Xu, L. Zhang, W. Lan, C. Zhou, Y. She and H. Fu, Food Chem., 2021, 346, 128923. 